Materials Science Forum, Vols. 426-432, 2003, pp. 35-42.

\title{
Advances in the Kinetic Theory of Carbide Precipitation
}

\author{
H. K. D. H. Bhadeshia \\ University of Cambridge \\ Department of Materials Science and Metallurgy \\ Pembroke Street, Cambridge CB2 3QZ, U.K., www.msm.cam.ac.uk/phase-trans
}

Keywords : Carbides, kinetics, steels, nucleation, growth, coarsening, microalloying, creep.

Abstract. The precipitation, coarsening and reversion of carbides can determine the quality of steels. This paper is a review of efforts towards better, practical theory for all of these solid-state reactions.

\section{Introduction}

Carbides are the essence of thermomechanically-processed microalloyed steels which are used in the hundreds of millions of tonnes in creating the infrastructure which leads to a better quality of life. Carbides also feature in special steels, such as the creep-resistant alloys which allow a greater efficiency in the generation of electricity. There is therefore a requirement to understand and model all aspects of carbides in the microstructure of iron and its alloys.

\section{Thermodynamics}

Thermodynamics forms the basis of any explanation of the properties of matter. In the present context it provides the framework for the calculation of multicomponent phase diagrams containing information about the equilibrium compositions and fractions of each of the co-existing phases. The traditional way of dealing with this in the context of microalloyed steels uses solubility products:

$$
\log \left\{\mathrm{w}_{\mathrm{Ti}_{\gamma}} \mathrm{w}_{\mathrm{C}_{\gamma}}\right\}=\frac{-7000}{T}+2.35 \quad \text { for the reaction } \quad \mathrm{Ti}_{\gamma}+\mathrm{C}_{\gamma} \rightleftharpoons \mathrm{TiC}
$$

where $T$ is the absolute temperature and the concentrations $(w)$ of titanium and carbon dissolved in austenite $(\gamma)$ are expressed in weight percent [1]. The origin of the solubility product is in the free energy change $\Delta G$ accompanying this reaction, with $\Delta G=0$ at equilibrium:

$$
\begin{gathered}
\Delta G=0=\Delta G^{0}+R T \ln \left\{\frac{a_{\mathrm{TiC}}}{a_{\mathrm{Ti}_{\gamma}} a_{\mathrm{C}_{\gamma}}}\right\} \text { and since } \Delta G^{0}=\Delta H^{0}-T \Delta S^{0} \\
\text { it follows that } \ln \left\{\mathrm{w}_{\mathrm{Ti}_{\gamma}} \mathrm{w}_{\mathrm{C}_{\gamma}}\right\}=\frac{-\Delta H^{0}}{R T}+\frac{\Delta S^{0}}{R} \quad \text { cf. equation } 1
\end{gathered}
$$

where $\Delta G^{0}$ is the standard free energy change; $\Delta H^{0}$ and $\Delta S^{0}$ the corresponding enthalpy and entropy changes respectively; $R$ is the gas constant; $a$ the activity and $x$ the mole fraction. Since the carbide is assumed here to be a pure, stoichiometric compound, $a_{\mathrm{TiC}}=1$; it is further assumed that $a \simeq x$. These approximations limit the applicability of solubility products. Kirkaldy and co-workers have generalised the products using Wagner interaction parameters to allow for the presence of many solutes and avoiding stoichiometry [2-4].

However, carbide equilibria are best dealt with using the CALPHAD method in which assessed thermodynamic data are used in combination with a free-energy minimisation algorithm to give the optimum estimates of multicomponent and multiphase diagrams [5]. Fig. 1 illustrates calculations using the CALPHAD method, for complex alloy steels containing many phases and components [6].

Kinetic factors often prevent transformations from occurring under equilibrium conditions; there are many different kinds of kinetically constrained equilibria that may arise naturally [7-11]. In the context of carbides, the transformation may occur by a mechanism which does not require the 


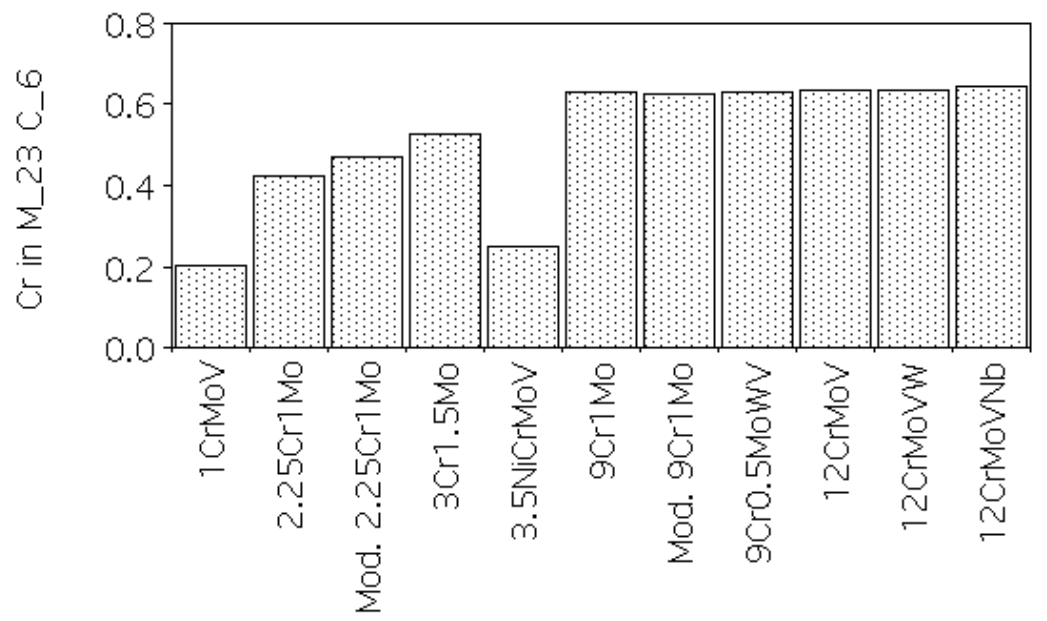

Fig. 1: The calculated chromium concentration (mole fraction) in $\mathrm{M}_{23} \mathrm{C}_{6}$ which is in equilibrium with ferrite, other carbides and intermetallic compounds, at $873 \mathrm{~K}$. The compositions of the steels listed on the horizontal axis are in [6].
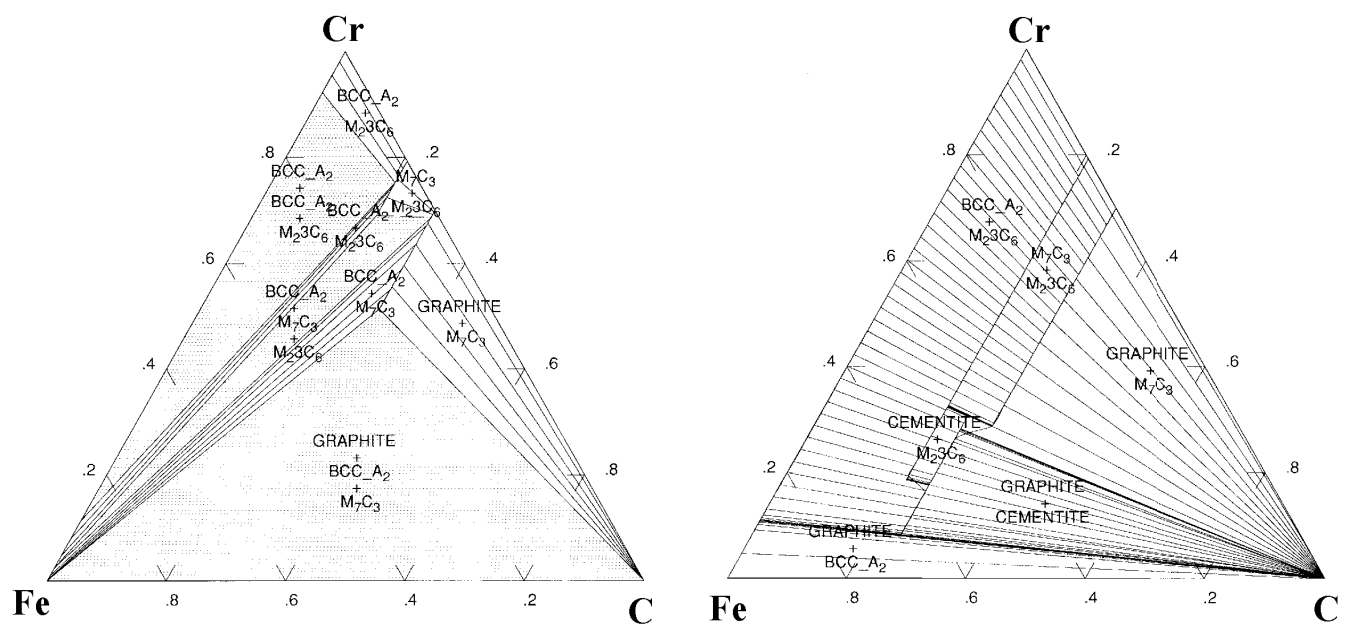

Fig. 2: Calculated isothermal $(700 \mathrm{~K})$ phase diagrams of the $\mathrm{Fe}-\mathrm{Cr}-\mathrm{C}$ system for the equilibrium and paraequilibrium scenarios.

diffusion of substitutional atoms. Hultgren [12-14] introduced the term 'paraequilibrium' to describe the constrained equilibrium between two phases which are forced to have the same substitutional to iron atom ratio, but which (subject to this constraint) achieve equilibrium with respect to carbon.

Fig. 2 shows the remarkable effect that such a constraint can have on the thermodynamics of carbide precipitation [15]. The tie-lines in the paraequilibrium diagram all radiate from the carbon corner of the plot because in this case the $\mathrm{Fe} / \mathrm{Cr}$ ratio must be constant for the carbide to be in paraequilibrium with the ferrite. The case illustrated is extreme in the sense that alloy carbides such as $\mathrm{M}_{23} \mathrm{C}_{6}$ and $\mathrm{M}_{7} \mathrm{C}_{3}$ can deviate from their equilibrium composition but in practice not to the extent illustrated [16]. Nevertheless, as discussed later in this paper, it is common for the ironbased carbides such as cementite to grow by paraequilibrium transformation. The equilibrium and paraequilibrium calculations illustrated in Fig. 2 were conducted using MTDATA [5], software which is available commercially from the National Physical Laboratory in the U.K.; it is exciting that phase 
diagram calculations can now be done routinely for constrained equilibria and hence can be more representative of reality.

One consequence of the fact that carbides may grow with a chemical composition which is inconsistent with equilibrium is that their composition may change towards equilibrium during service at elevated temperatures [17-19]. This phenomenon is exploited in the assessment of remaining life in power plant components [20].

\section{Nucleation}

The theoretical framework for the process of nucleation [21] is probably well-founded but it contains terms which are exceedingly difficult to predict or even to measure properly. We shall focus here on the difficulties rather than reproduce standard nucleation theory. The most important of the problem parameters are the interfacial energy per unit area $\sigma$, the strain energy per unit volume $E$ and the number density of nucleation sites $N_{V}$.

It has been know for some time that the interfacial energy between a droplet and its vapour must depend on the droplet size $[21,22]$. When dealing with solid-state transformations $\sigma$ can be expected to be both orientation and size dependent. Solid-state transformations also led to strains and the accompanying strain energy $E$ must be accounted for. A combination of the orientation dependence of $\sigma$ and the need to minimise $E$ then determines the shape of the nucleus, which itself must be size dependent. In the case of heterogeneous nucleation the shape must also depend on the interaction with the nucleating defect.

These complications are far too difficult to deal with when considering practical materials; $\sigma$ inevitably is deduced empirically by fitting rate data to elementary models of nucleation. There is a saving grace when dealing with sequences of precipitation reactions in which a number of metastable phases precede the equilibrium precipitate. The metastable phases occur only because they are easier to nucleate and hence their interfacial energies during nucleation must be smaller than that of the ultimate precipitate. The observed sequence can therefore be used to rank interfacial energies [23].

Nucleation is frequently heterogeneous; it occurs at defects such a vacancies, stacking faults, dislocations, dislocation-clusters (e.g. shear bands), twin boundaries, grain boundaries and inclusions. The number density of the defects is a function of the processing history and can be very difficult to estimate from the processing parameters. Therefore, $N_{V}$ is usually a measured parameter; for

example, when nucleation occurs at grain boundaries, $N_{V} \propto \bar{L}^{-1}$ where $\bar{L}$ is the mean lineal intercept defining the grain size. Even this approach is vague because it fails to account for structure of the boundary; the potency of a grain boundary as a nucleation site is in practice a strong function of its crystallographic character [24-26].

Another common approximation in nucleation theory involves the neglect of transient effects, i.e. it is assumed that the nucleation rate rapidly reaches a steady-state value. This may be reasonable when the nucleus relaxation time is much less than that for transformation [27].

\section{Growth}

Many attempts at modelling carbide growth assume a binary approach in which only one of the solutes is considered in the growth equations, thereby violating local equilibrium at the interface. This procedure is often falsely justified by stating that the solute considered is the one that controls transformation. In fact, the theory for diffusion in multicomponent systems is well established $[28,29,11]$. We shall attempt here to illustrate in a simple way why this should be applied when modelling carbides in ternary or higher order systems.

For isothermal transformation in a binary alloy, the concentrations at the interface are given by a tie-lie of the phase diagram as shown in Fig. 3. The diffusion flux of solute from the interface must equal the rate at which solute is incorporated in the precipitate so that:

$$
\underbrace{\left(c^{\theta \gamma}-c^{\gamma \theta}\right) \frac{\partial z^{*}}{\partial t}}_{\text {rate solute partitioned }}=\underbrace{-\left.D \frac{\partial c}{\partial z}\right|_{z=z^{*}}}_{\text {diffusion flux to interface }}
$$

where $z$ is a coordinate normal to the interface with a value $z^{*}$ at the position of the interface. Note 

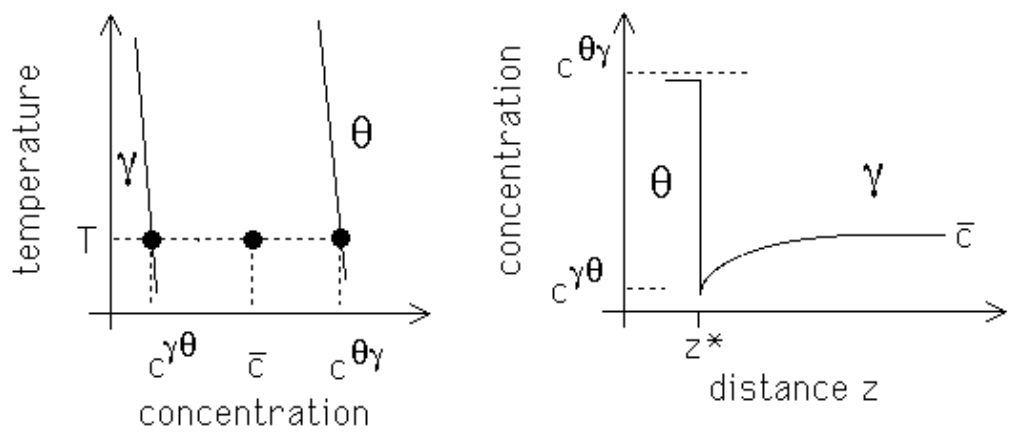

Fig. 3: Phase diagram and its relationship to the concentration profile at the carbide $(\theta)$ - matrix $(\gamma)$ interface during diffusion-controlled growth.

that the concentration gradient is evaluated at the position of the interface $\left(z=z^{*}\right)$. D is the solute diffusivity.

Consider now a ternary steel, say $\mathrm{Fe}-\mathrm{X}-\mathrm{C}$, where the $X$ stands for a substitutional solute. It would be necessary to satisfy two equations of the form of equation 3 , simultaneously, for each of the solutes [30-32]:

$$
\left.\begin{array}{rl}
\left(c_{1}^{\theta \gamma}-c_{1}^{\gamma \theta}\right) v & =-D_{1} \nabla c_{1} \\
\left(c_{2}^{\theta \gamma}-c_{2}^{\gamma \theta}\right) v & =-D_{2} \nabla c_{2}
\end{array}\right\}
$$

where the subscripts refer to the solutes ( 1 for carbon and 2 for $X$ ). The interface velocity $v=\partial z^{*} / \partial t$.

Because $D_{1} \gg D_{2}$, these equations cannot in general be simultaneously satisfied for the tie-line passing through the alloy composition $\bar{c}_{1}, \bar{c}_{2}$. However, in a ternary alloy, there are many tie-lines to choose from because of the extra degree of freedom granted by the phase rule. There is an entire phase field in which the carbide and matrix can exist in equilibrium so it is possible to find another tie line which satisfies these equations simultaneously. The important point is that the correct tie line will not in general pass through the average composition $\bar{c}_{1}, \bar{c}_{2}$. This is where the application of binary theory to a ternary or multicomponent problem goes wrong. Expressed in another way, the equations must be solved in such a way that the variety of solute fluxes can all keep pace with the interface whilst maintaining local equilibrium at the interface.

The discussion so far has assumed a flat interface; precipitates tend to be in the form of spheres, needles or plates. The curvature of the interface then leads to a change in equilibrium, the famous capillarity effect. There is only an approximate solution which accounts for capillarity during the growth of spheres [33]. Diffusion-controlled growth follows a parabolic relationship in which the particle radius $\rho=\alpha(D t)^{0.5}$ where $\alpha$ is a one-dimensional parabolic rate constant. For a small supersaturation $\Omega=\left(\bar{c}-c^{\gamma \theta}\right) /\left(c^{\theta \gamma}-c^{\gamma \theta}\right)$,

$$
\alpha=\sqrt{2 \Omega\left[1-\frac{\rho_{C}}{\rho}\right] /\left[1-\Omega \frac{\rho_{C}}{\rho}\right]} \quad \text { and } \quad \Omega \ll 1
$$

where $\rho_{C}$ is the critical radius below which capillarity prevents growth.

The theory for needle and plate growth with capillarity and for a multicomponent system becomes complicated but is tractable; it has been described elsewhere [33-35].

\section{Overall Kinetics}

The carbide precipitation processes considered here all thermodynamically of first order in the Ehrenfest classification [36]; the formation of each particle therefore requires nucleation in the classical sense and subsequent growth. We assume here that nucleation and growth phenomena are fully understood, to enable the evolution of volume fraction of transformation to be calculated.

The estimation of volume fraction requires an acknowledgment of the possibility of impingement between particles growing from different sites. This is done using the extended volume concept of 
Johnson, Mehl, Avrami, and Kolmogorov [21] as illustrated in Fig. 4. Suppose that two particles exist at time $t$; a small interval $\delta t$ later, new regions marked $a, b, c \& d$ are formed assuming that they are able to grow unrestricted in extended space whether or not the region into which they grow is already transformed. However, only those components of $a, b, c \& d$ which lie in previously untransformed matrix can contribute to a change in the real volume of the product phase (identified by the subscript ' 1 ') so that :

$$
d V_{1}=\left(1-\frac{V_{1}}{V}\right) d V_{1}^{e}
$$

where it is assumed that the microstructure develops randomly. The superscript $e$ refers to extended volume, $V_{1}$ is the volume of phase 1 and $V$ is the total volume. Multiplying the change in extended volume by the probability of finding untransformed regions has the effect of excluding regions such as $b$, which clearly cannot contribute to the real change in volume of the product. The extended volume term is easy to calculate from the nucleation and growth rates, because it neglects impingement but it is the real volume that is needed. This requires the equation to be integrated to obtain the real volume fraction,

$$
\frac{V_{1}}{V}=1-\exp \left\{-\frac{V_{1}^{e}}{V}\right\}
$$
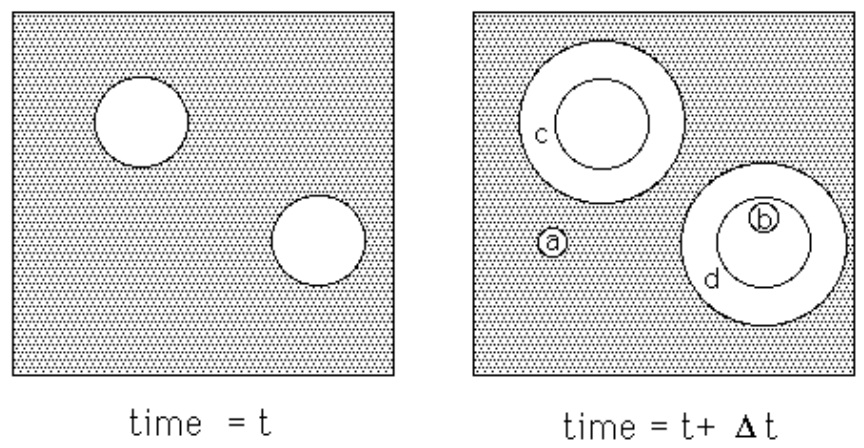

Fig. 4: The concept of extended volume. Two precipitate particles have nucleated and grown to a finite size in the time $t$. New regions $c$ and $d$ are formed as the original particles grow, but $a \& b$ are new particles, of which $b$ is unreal because it has formed in a region which is already transformed.

\section{Overall Kinetics: Simultaneous Transformations}

Many of the precipitates found in power plant steels have crystal structures and compositions which are quite different from those of the ferrite matrix. The precipitate/matrix interfacial energy can therefore be expected to be large, making it difficult for the equilibrium precipitate to nucleate. Consequently, decomposition often starts with the formation of one or more metastable phases which are kinetically favoured. These must eventually dissolve as equilibrium is approached. It follows that many precipitation and dissolution reactions may occur simultaneously.

When more than one reaction occurs at the same time, they interfere with each other in a way which is seminal to the development of power plant microstructures. It is not possible to deal with more than one reaction at time using ordinary Avrami theory, but a recent adaptation has generalised the theory for simultaneous reactions $[23,37-40]$. When phases $\beta$ and $\theta$ precipitate at the same time from a parent phase $\theta$ equation 5 is replaced by a pair of coupled equations as follows:

$$
d V_{\beta}=\left(1-\frac{V_{\beta}+V_{\theta}}{V}\right) d V_{\beta}^{e}
$$


and similarly for $\theta$,

$$
d V_{\theta}=\left(1-\frac{V_{\beta}+V_{\theta}}{V}\right) d V_{\theta}^{e}
$$

Since $V_{\beta}$ is in general expected to be some complicated function of $V_{\theta}$, numerical integration is used and exploited to change the boundary conditions for nucleation and growth during the course of transformation, thus accounting for the change in the matrix composition using a mean field approximation. The method can in principle be applied to any number of simultaneous reactions with a corresponding set of coupled equations, and indeed has been used widely in the modelling of complex carbide-precipitation reactions [41-44]. Fig. 5 shows the kind of calculations that can be done [45].

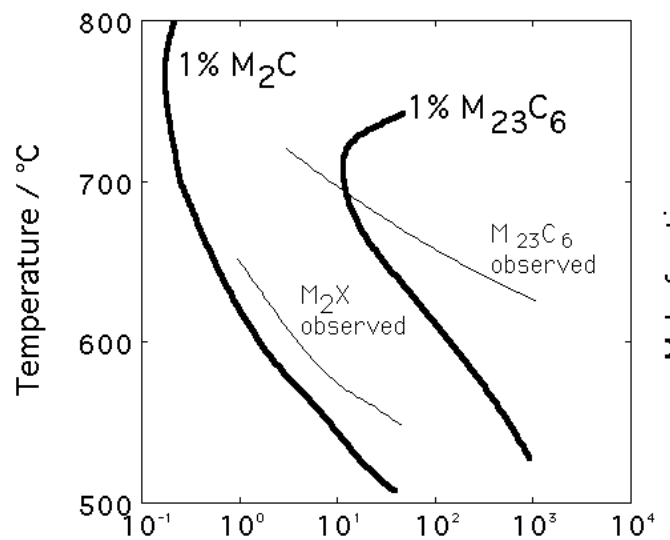

(a)
Time / $\mathrm{h}$

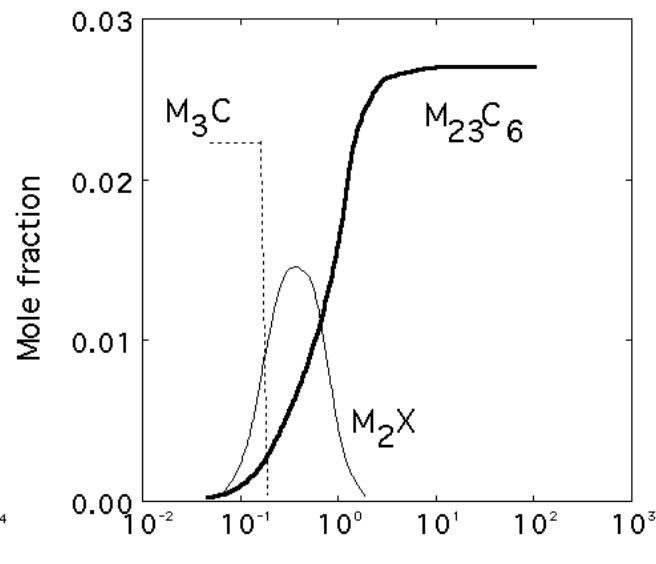

(b)

Time / h

Fig. 5: Precipitation kinetics in a 2.25Cr1Mo steel. (a) Comparison of calculated and measured data. (b) Calculated isothermal transformation curves for precipitation in a novel $10 \mathrm{CrMoW}$ alloy at $600^{\circ} \mathrm{C}$.
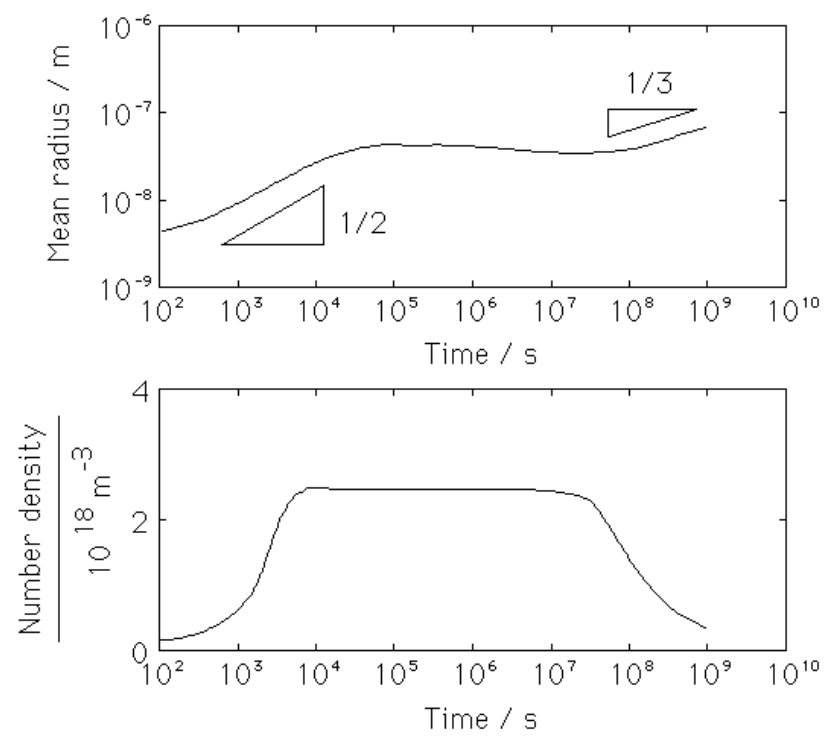

Fig. 6: The precipitation and coarsening of niobium carbide precipitates in austenite. Details can be found in [45]. 


\section{Coarsening}

Coarsening kinetics do not require an explicit treatment but follow naturally from the precipitation theory which for each phase includes the capillarity effect. Indeed, it is well known that the separation of precipitation and coarsening is artificial, because capillarity makes the smaller precipitates grow at a slower rate than those which nucleated first and hence are larger, even before the equilibrium fraction is reached. Fig. 6 shows the results of calculations of this kind, the mean particle radius at first increasing approximately parabolically with time as all particles grow from solid solution [46]. The mean radius then changes as about $t^{\frac{1}{3}}$ at longer times as the number density of particles decreases, consistent with expectations from coarsening theory. It is important to emphasise that there is no separate treatment of precipitation and coarsening here, both are in an inclusive theory.

The difficulties associated with the modelling of coarsening in multiphase systems have been described elsewhere [6].

\section{Summary}

It is in the treatment of carbide precipitation in complex systems that there has been greatest progress, both theoretically and experimentally. By complex we mean multicomponent and multiphase alloys, of the kind common in industry. Progress in the following areas has been particularly potent:

(i) The ability to deal routinely with the simultaneous diffusion of many solutes, even when the solutes have enormously disparate diffusivities.

(ii) Mathematical solutions now exist for the diffusion-controlled growth of a variety of shapes (spheres, needles, plates) in multicomponent systems.

(iii) It is now possible to generalise Avrami theory to handle many precipitation reactions occurring at the same time.

The following areas require further work:

(i) Nucleation theory requires many empirical inputs and hence is difficult to use in a predictive sense.

(ii) Coarsening theory for multiphase reactions.

Notwithstanding the difficulties outlined above, the methods have been successfully applied in alloy design but their full potential has yet to be exploited. The subject is one of the most exciting in the field of materials science.

\section{Acknowledgments}

I am very grateful to the organising committee of THERMEC 2003 and to Professor Chandra for the opportunity to present this work. I am extremely conscious of the help I have received from many students and friends, whose contributions can be seen on www.msm.cam.ac.uk/phase-trans.

\section{References}

[1] J. C. Herman, P. Messien \& T. Greday: Thermomechanical Processing of Microalloyed Austenite, eds. A. DeArdo, G. Ratz and P. Wray, TMS-AIME, Pennsylvania, USA, (1981) 655.

[2] R. C. Sharma, V. K. Lakshmanan and J. S. Kirkaldy: Metall. Trans. 15A (1984) 545.

[3] V. K. Lakshmanan and J. S. Kirkaldy: Metall. Trans. 15A (1984) 541.

[4] K. Balasubramanian and J. S. Kirkaldy: CALPHAD 9 (1985) 103.

[5] K. Hack: Thermodynamics at Work, Institute of Materials, London. (1996)

[6] H. K. D. H. Bhadeshia: ISIJ International 41 (2001) 626.

[7] J. W. Gibbs: The Scientific Papers of J.W. Gibbs, Dover Publications, New York (1961) 57.

[8] L. S. Darken: TMS-AIME 180 (1949) 430.

[9] L. S. Darken and R. W. Gurry: Physical Chemistry of Metals, McGraw-Hill, New York (1953)

[10] J. C. Baker and J. W. Cahn: Solidification, ASM, Ohio (1971) 23.

[11] H. K. D. H. Bhadeshia: Progress in Materials Science 29 (1985) 321.

[12] A. Hultgren: Jernkontorets Ann. 135 (1951) 403.

[13] M. Hillert: Report, Swedish Institute for Metals Research, Sweden (1953) 
[14] E. Rudberg: Jernkontorets Ann. 136 (1952) 91.

[15] J. D. Robson and H. K. D. H. Bhadeshia: Microstructural development and stability in high chromium ferritic power plant steels, eds. A. Strang and D. J. Gooch, Institute of Materials, London (1997) 179.

[16] A. Strang, V. Vodarek and H. K. D. H. Bhadeshia: Modelling of Microstructural Evolution in Creep Resistant Materials, eds A. Strang and M. McLean, Institute of Materials, London (1999) 129.

[17] R. B. Carruthers and M. J. Collins: Metal Science 17 (1983) 107.

[18] A. Afrouz, M. J. Collins and R. Pilkington: Metal Technology 10 (1983) 461.

[19] H. K. D. H. Bhadeshia: Materials Science and Technology 5 (1989) 131.

[20] H. K. D. H. Bhadeshia, A. Strang and D. J. Gooch: Int. Materials Reviews 43 (1998) 45.

[21] J. W. Christian: Theory of Transformations in Metals and Alloys, Pergamon Press, Oxford, 2nd edition (1975)

[22] G. C. Benson and R. Shuttleworth: J. Chem. Phys. 19 (1951) 130.

[23] J. D. Robson and H. K. D. H. Bhadeshia: Mat. Sci. Tech. 13 (1997) 631.

[24] H. U. Hong, B. S. Rho and S. W. Nam: Materials Science and Engineering A318 (2001) 285.

[25] M. Shimada, H. Kokawa, Z. J. Wang, Y. S. Sato and I. Karibe: Acta Materialia 50 (2002) 2331.

[26] D. N. Wasnik, V. Kain, I. Samajdar, B. Verlinden and P. K. De: Acta Materialia 50 (2002) 4587 .

[27] M. D. Demetriou, N. M. Ghoniem and A. S. Lavine: J. Chem. Phys. 117 (2002) 10739.

[28] L. Onsager: Ann. N. Y. Acad. Sci. 46 (1945-1946) 241.

[29] J. S. Kirkaldy: Adv. Mater. Res. 4 (1970) 55.

[30] D. E. Coates: Metall. Trans. 4 (1973) 395.

[31] D. E. Coates: Metall. Trans. 4 (1973) 1077.

[32] D. E. Coates: Metall. Trans. 4 (1973) 2313.

[33] P. E. J. Rivera-Diaz-del-Castillo and H. K. D. H. Bhadeshia: Materials Science and Technology 17 (2001) 30.

[34] P. E. J. Rivera-Diaz-del-Castillo and H. K. D. H. Bhadeshia: Materials Science and Technology 17 (2001) 25.

[35] P. E. J. Rivera-Diaz-del-Castillo and H. K. D. H. Bhadeshia: Metallurgical and Materials Transactions 33A (2002) 1075.

[36] P. Ehrenfest: Proc. Acad. Sci. Amsterdam 36 (1933) 153.

[37] H. K. D. H. Bhadeshia: Proc. of Solid-Solid Phase Transformations '99, eds M. Koiwa, K. Otsuka and T. Miyazaki, Japan Institute for Metals, Kyoto, Japan (1999) 1445.

[38] S. J. Jones and H. K. D. H. Bhadeshia: Acta Materialia 45 (1997) 2911.

[39] S. J. Jones and H. K. D. H. Bhadeshia: Metallurgical and Materials Trans. 28A (1997) 2005.

[40] T. Kasuya, K. Ichikawa, M. Fuji and H. Bhadeshia: Materials Sci. ES Techn. 15 (1999) 471.

[41] J. D. Robson and H. K. D. H. Bhadeshia: Mat. Sci. Tech. 13 (1997) 631.

[42] J. D. Robson and H. K. D. H. Bhadeshia: Calphad 20 (1996) 447.

[43] N. Fujita and H. K. D. H. Bhadeshia: ISIJ International 42 (2002) 760.

[44] N. Fujita and H. K. D. H. Bhadeshia: Materials Science and Technology 17 (2001) 403.

[45] N. Fujita and H. K. D. H. Bhadeshia: Advanced Heat Resistant Steels for Power Generation, San Sebastian, Institute of Materials, London (1998) 223.

[46] F. Brun, T. Yoshida, J. D. Robson, V. Narayan, H. K. D. H. Bhadeshia and D. J. C. MacKay: Materials Science and Technology 15 (1999) 547.

[47] N. Fujita, H. K. D. H. Bhadeshia and M. Kikuchi: Metallurgical and Materials Transactions 33A (2002) 3339. 